\title{
A double droplet trap system for studying mass transport across a droplet-droplet interface $\dagger$
}

\author{
Yunpeng Bai, ${ }^{a}$ Ximin He, ${ }^{a}$ Dingsheng Liu, ${ }^{a}$ Santoshkumar N. Patil, ${ }^{b}$ Dan Bratton, ${ }^{a}$ Ansgar Huebner, ${ }^{c}$ \\ Florian Hollfelder, ${ }^{c}$ Chris Abell ${ }^{b}$ and Wilhelm T. S. Huck ${ }^{* a d}$
}

\author{
Received 30th November 2009, Accepted 22nd January 2010 \\ First published as an Advance Article on the web 16th February 2010 \\ DOI: 10.1039/b925133b
}

\begin{abstract}
Here we present the design, fabrication and operation of a microfluidic device to trap droplets in a large array of droplet pairs in a controlled manner with the aim of studying the transport of small molecules across the resultant surfactant bilayers formed between the droplet pairs.
\end{abstract}

\section{Introduction}

Recent advances in droplet-based microfluidic systems have demonstrated the suitability of the microdroplet platform for the study of chemical and biochemical reactions in moving or stationary droplets. ${ }^{1-6}$ In these studies, droplets are usually considered as self-contained microreactors that prevent sample diffusion and cross-contamination. However, this assumption might not be valid in every experiment, as the surfactant layers around microdroplets are in principle permeable to small molecules. $^{7}$ Bayley and co-workers studied the formation of 'droplet interface bilayers' between droplets in oil using phospholipids as surfactants. The incorporation of membrane proteins into the interface bilayers allowed the construction of droplet networks with complex properties. ${ }^{8-10}$ These experiments were performed using small numbers (tens) of large, $700 \mu \mathrm{m}$ diameter, $200 \mathrm{~nL}$ droplets. Here, we introduce a microfluidic device in which thousands of droplet-droplet interfaces and the transfer across these interfaces can be studied. The droplet interfaces in our studies consist of swollen polymer brush bilayers (in contrast to lipid bilayers) due to the nature of the surfactants typically used in microdroplets in microfluidics experiments. The potential for a systematic study of the transport across such interfaces will be of importance in, for example, protein crystallization in microdroplets, ${ }^{11}$ and experiments involving close-packed arrays of microdroplets in microfluidics devices. ${ }^{7,12}$ Previous work has established robust procedures for forming, ${ }^{13}$ dividing,,${ }^{14}$ fusing, ${ }^{15}$ interrogating, ${ }^{16}$ and sorting droplets, ${ }^{17}$ as well as storing or trapping droplets on-chip. ${ }^{18-20}$ However, in most cases, dropletdroplet interactions have not been studied in detail. The inchannel array of traps shown in Fig. 1 were designed to provide a simple and effective strategy for trapping droplets pairs and

${ }^{a}$ Melville Laboratory for Polymer Synthesis, Department of Chemistry, University of Cambridge, Lensfield Road, Cambridge, UK CB2 1EW. E-mail:wtsh2@cam.ac.uk; Tel: +441223 334370

${ }^{b}$ Department of Chemistry, University of Cambridge, Lensfield Road, Cambridge, UK CB2 1EW

'Department of Biochemistry, University of Cambridge, 80 Tennis Court Road, Cambridge, UK CB2 1GA

${ }^{d}$ Radboud University Nijmegen, Institute for Molecules and Materials, Heyendaalseweg 135, 6525 AJ Nijmegen, The Netherlands

$\dagger$ Electronic supplementary information (ESI) available: Supplementary figures. See DOI: 10.1039/b925133b study the transfer of molecules across droplet-droplet interfaces. By loading the traps in two flow directions, $73 \%$ (out of 1500) droplet pairs were $\mathrm{AB}$ (rather than $\mathrm{AA}$ or $\mathrm{BB}$ ), significantly increasing the odds over a statistical $50 \% \mathrm{AB}$ occupancy when both droplets originate from one inlet. Upon arraying of the heterogeneous droplet pairs, transport phenomena, in this case of fluorescent dye molecules and hydrogen peroxide, across the surfactant bilayers can be studied.

\section{Materials and methods}

\section{Materials}

Poly(dimethylsiloxane) (PDMS, Sylgard 184) was obtained from Dow Corning (UK). FC-77, resorufin, horseradish peroxidase, mineral oil, and Span80 were purchased from Sigma-Aldrich. Hydrogen peroxide (Breckland Scientific Supplies) solutions of different concentrations were prepared freshly using $\mathrm{pH}$ 8.0 Tris$\mathrm{HCl}$ buffer before each experiment. EA surfactant (RAS 168069) was obtained from RainDance Technologies.

\section{Microfluidic device fabrication}

The microfluidic device used in this paper was fabricated in PDMS using standard soft lithographic methods. ${ }^{21,22}$ Briefly, SU8-2025 photoresist (MicroChem) was spin-coated to a final film thickness of $50 \mu \mathrm{m}$, as measured by profilometry on the finished master (DekTak 150). After spinning, the wafer was prebaked $\left(3 \mathrm{~min}\right.$ at $65^{\circ} \mathrm{C}$, then $6 \mathrm{~min}$ at $95^{\circ} \mathrm{C}$ and finally $3 \mathrm{~min}$ at $65^{\circ} \mathrm{C}$ ), and then exposed to UV light through a dark-field mask (Circuitgraphics) on a mask aligner (MJB4, Suss Microtech). After postbaking for $1 \mathrm{~min}$ at $65{ }^{\circ} \mathrm{C}$ and $3 \mathrm{~min}$ at $95{ }^{\circ} \mathrm{C}$, the master was developed for $6 \mathrm{~min}$ and then hard-baked for $1 \mathrm{~min}$ at $170{ }^{\circ} \mathrm{C}$. PDMS prepolymer and curing agent (10: 1, Sylgard 184) was poured over the master, degassed for $30 \mathrm{~min}$ and then baked overnight at $75{ }^{\circ} \mathrm{C}$. The devices were cut and peeled off the master. Access holes for the inlet tubes were punched using a biopsy punch. The PDMS was then exposed to an air plasma for $8 \mathrm{~s}$ (Diener Femto plasma asher), sealed to a glass microscope slide, and baked overnight at $75^{\circ} \mathrm{C}$. For fluorophilic surfaces, the channels of the device were treated with Aquapel agent and then FC-77. To avoid evaporation of the aqueous phase the PDMS device was sealed in a chamber filled with water for 3 days before performing experiments. 

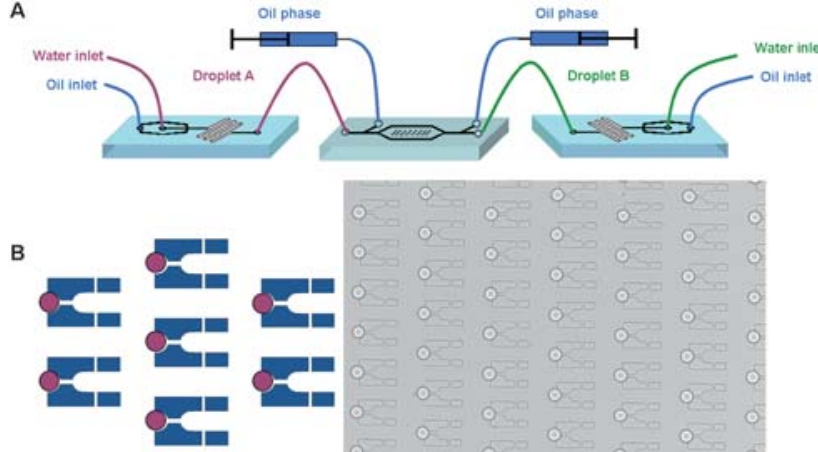

C

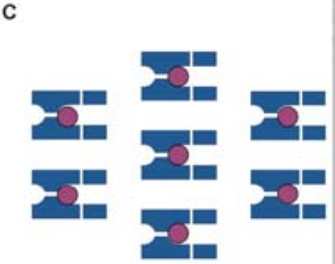

D
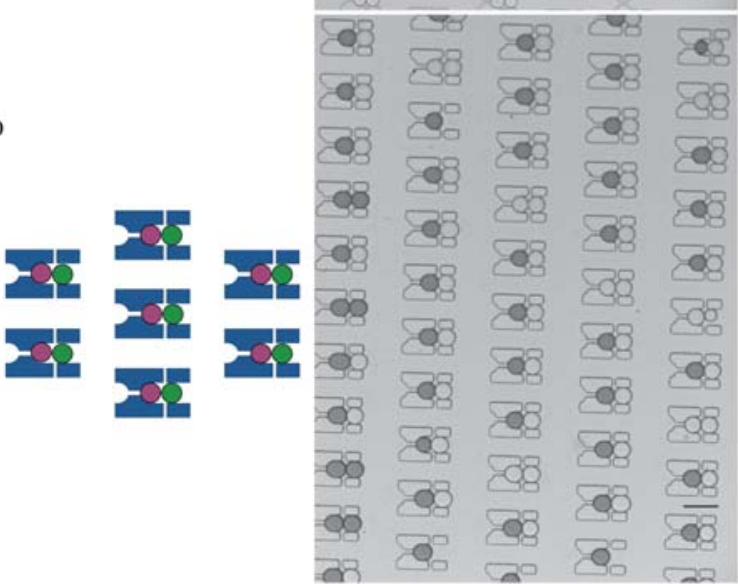

Fig. 1 (A) Droplets were generated by flow-focusing in the left device, and injected into the right device through tubing. FC-77 and 1\% RainDance EA surfactant was used as the oil phase. (B) Droplets were first loaded toward the back-side cup of traps. Bright-field microscopy images give the whole picture and details of trapped droplets (right). (C) The direction of the flow was reversed, and the droplets were transferred down into the front-side capture cup two rows below. (D) The second droplet was loaded from the top, and captured in front of the first droplet type. To discriminate the two droplets, the first droplet contains a black food dye and the second one is empty. Pictures of (B), (C) were taken from one experiment, and (D) from another experiment. Scale bars: (B), (C) and (D) $120 \mu \mathrm{m}$.

\section{Operation of the device}

The flow was driven with Harvard Apparatus 2000 syringe infusion pumps using plastic syringes (B. Braun, Germany) connected to polyethylene tubing (Beckman and Dickinson, U. S.). The mixture of FC-77/1\% RainDance EA surfactant $(\mathrm{w} / \mathrm{w} \%)$ was used as the oil phase in a fluorophilic PDMS device. Flow-focusing configuration was used to generate $50 \mu \mathrm{m}$ sized droplets. The size, frequency and speed of the droplets within the device were regulated by controlling the flow rate of the aqueous solutions and the oil flow. A typical setup is the combination of
$60 \mu \mathrm{L} \mathrm{h}^{-1}$ oil flow and $20 \mu \mathrm{L} \mathrm{h}^{-1}$ aqueous flow. These droplets were injected into another device for further trapping. All the experiments were conducted at $24{ }^{\circ} \mathrm{C}$.

\section{Detection systems}

A Phantom V72 camera recorded pictures at 1000 frames per second in bright-field mode and was used to observe the trapped droplets in microfluidic devices. Imaging of the fluorescence within a chamber was performed using an EM-CCD camera (Xion+, Andor Technologies) connected to an inverted microscope (IX71, Olympus) operating in epifluorescence mode, with a mercury lamp (U-LH100HG, Olympus) for wide-field illumination and appropriate filters (U-MWIG3,Olympus) to separate the fluorescence excitation and emission light. A computercontrolled shutter was added to the excitation path to limit the time during which excitation light was incident upon the sample in order to minimize photobleaching. To observe the whole chamber, an automatic microscope stage (ProScan II, Prior Scientifc) was fitted to the microscope. The measurement of fluorescence intensity was performed using Labview software, and the analysis of the gray value of pictures was carried out using Image $\mathbf{J}$.

\section{Results and discussion}

Our devices consist of two separate chips to generate and trap droplets, respectively. The first chip incorporates a flow-focussing geometry to generate water-in-oil (fluorous oil FC-77, Raindance EA surfactant) droplets with a diameter of $50 \mu \mathrm{m}$ (Fig. 1A). ${ }^{23}$ Droplet size and frequency were controlled by a combination of channel dimensions and flow rates. By flowing the droplets through a long winding channel (residence time $\sim 10$ s), a surfactant monolayer was allowed to form around the droplets before they entered the outlet tubing. ${ }^{24}$ Once a stable flow of droplets was obtained, the tubing was connected to the trapping chip. Visual inspection confirmed that the passage of droplets through the tubing (which has a larger internal diameter than the channel cross-section) did not lead to droplet fusion. To circumvent the problem of random filling of the traps, we modified a recently published strategy for trapping two different cells in a microfluidic device. ${ }^{25}$ First, droplets were trapped in shallow 'wells' formed by PDMS posts with a narrow gap that was significantly smaller than the droplet diameter (Fig. 1B). As shown recently by Huebner et al., ${ }^{18}$ as soon as droplets blocked the central exit the carrier fluid flow was forced around the droplet/PDMS barrier, thereby trapping the droplets. Subsequently, the flow direction was reversed by connecting the second droplet generating chip at the other side of the trapping device. By reversing the flow, the droplets present in the traps were moved into the deeper traps (Fig. 1C). Then, the second type of droplets were allowed to enter the device from the opposite direction as the first droplets, and these droplets then formed pairs with the previously trapped ones (Fig. 1D). An optimized design of columns with a spacing of $60 \mu \mathrm{m}$ and row spacing of $120 \mu \mathrm{m}$, resulted in the formation of approximately $73 \%$ of $\mathrm{AB}$ droplet pairs, $7 \%$ AA or $7 \%$ BB pairs, and $13 \%$ empty traps in 1500 traps. Droplet pairs were stable for at least $6 \mathrm{~h}$ and no changes in droplet volumes were observed during this time. 
Traps filled with droplets containing $50 \mu \mathrm{M}$ fluorescent dye (resorufin) showed a fluorescence intensity variation from droplet to droplet of less than $4 \%$ in the $x$-direction and $10 \%$ in the $y$-direction respectively (ESI Fig. SI $1 \dagger$ ).

With a robust method for forming large numbers of droplet pairs in place, we designed a model experiment for studying the diffusion of small molecules from one droplet to another. Previous work by Bayley and co-workers studied the diffusion of ions across membrane protein-containing lipid bilayers formed between two aqueous droplets in hexadecane. ${ }^{8-10}$ We previously established the leakage of hydrophobic small molecules out of droplets into the surrounding oil and PDMS; a phenomenon that can be slowed down by the choice of surfactant or by coating the interior of side of the water-oil interface. ${ }^{7}$

Droplet pairs trapped on-chip show a clear deformation in the region where the droplets are touching. This is indicative of a draining of the oil between the droplets in that region (Fig. 2). ${ }^{26,27}$ By increasing the oil flow rate four-fold, the deformation of the droplets becomes more pronounced and the length of the interface increases by approximately $40 \%$ (as measured in the images in Fig. 2A and D). The interface formed between two aqueous droplets stabilized with a polymeric surfactant and, after drainage of the oil phase, is essentially a swollen polymer brush bilayer.

First, we used a common fluorescent dye (fluorescein) as an indicator to study the diffusion of small molecules across the surfactant bilayer between two droplets (Fig. 3). The results indicates that the nature of the bilayer determines the transfer rate of molecules. For the $1 \% \operatorname{Span} 80 /$ mineral oil system, the fluorescence intensity increased significantly during a $10 \mathrm{~h}$ measurement (Fig. 3A). However, no obvious increase in fluorescence intensity was observed for $1 \%$ RainDance EA surfactant/FC-77 system (Fig. 3B). These results corroborate previous reports that $\operatorname{Span} 80$ has a high fluidity at the water/oil interface and shows high leakage of entrapped water-soluble dye molecules. $^{28}$ In contrast, the RainDance surfactant leads to more stable droplets and provides a superior 'sealing' of the interface allowing dye-tagged drug screening in droplets. ${ }^{3}$

In a number of recent studies, microdroplets have been used as isolated containers for studying the kinetics of

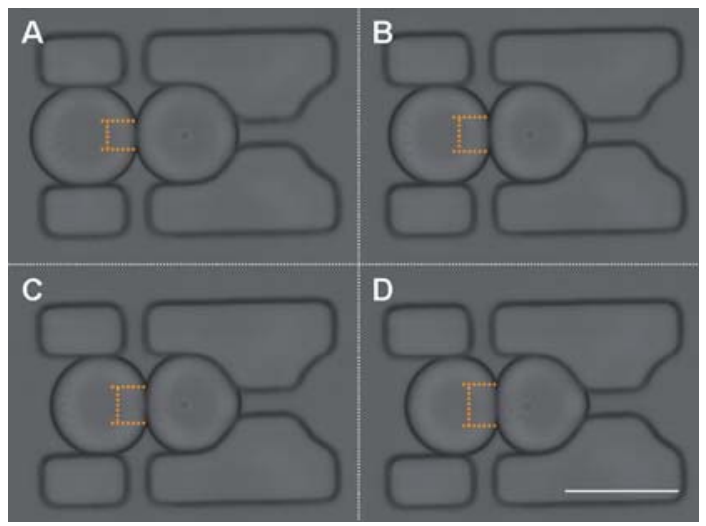

Fig. 2 Two droplets were trapped by different oil flow rates: (A) $2000 \mu \mathrm{L}$ $\mathrm{h}^{-1}$, (B) $4000 \mu \mathrm{L} \mathrm{h}^{-1}$, (C) $6000 \mu \mathrm{L} \mathrm{h}^{-1}$ and (D) $8000 \mu \mathrm{L} \mathrm{h}^{-1}$. Orange lines indicate the length of the interface. Scale bar: $60 \mu \mathrm{m}$. 1\% RainDance EA surfactant/FC-77.

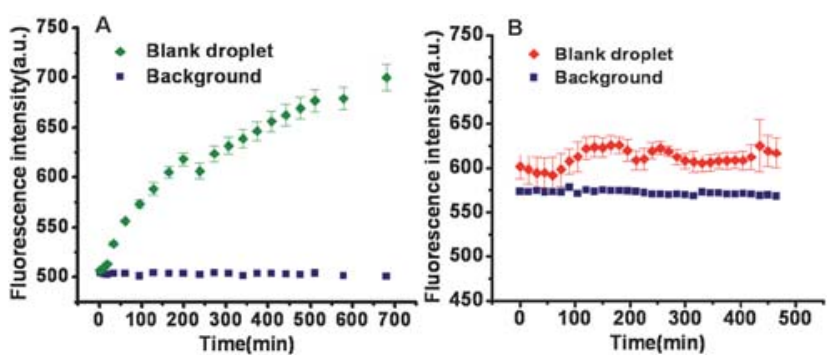

Fig. 3 The transfer of $50 \mu \mathrm{M}$ fluorescein across two different surfactant bilayers: (A) 1\% Span80/mineral oil and (B) 1\% RainDance EA surfactant/FC-77. Blank droplet indicates the droplet without fluorescence at time zero trapped with another droplet containing $50 \mu \mathrm{M}$ fluorescein.

compartmentalized enzymes or cells. ${ }^{4,5}$ Recently, more complex fluidic experiments have attempted to mimic fundamental biological processes such as quorum sensing and cell-cell communication by allowing diffusion of solutes between microfabricated compartments. ${ }^{29-32}$ In order to investigate the potential of the trapping device, a simple diffusion-reaction model system was designed based on the enzymatic reactions of $\mathrm{H}_{2} \mathrm{O}_{2}$ and horseradish peroxidase (HRP) to oxidise a fluorescent substrate resorufin to a non-fluorescent product resazurin (Fig. 4A) ${ }^{33}$ Droplet pairs in the traps contained either a mixture of $\mathrm{pH} 8.0$ Tris- $\mathrm{HCl}$ solution of resorufin $(50 \mu \mathrm{M})$ and horseradish peroxidase $\left(0.07 \mathrm{mg} \mathrm{mL}^{-1}\right)$, or a solution of $0.3 \mathrm{M} \mathrm{H}_{2} \mathrm{O}_{2}$ in $\mathrm{pH}$ 8.0 Tris- $\mathrm{HCl}$ buffer. The reaction solution was adjusted to $\mathrm{pH} 8.0$ during the experiments to prevent spontaneous decomposition of resorufin. ${ }^{34}$

As soon as the two droplets were pressed into contact in the traps and an interface was established, hydrogen peroxide started to diffuse across the interface due to the concentration difference between two droplets. As the product of the enzymatic reaction, resazurin, is non-fluorescent, the fluorescence intensity of the
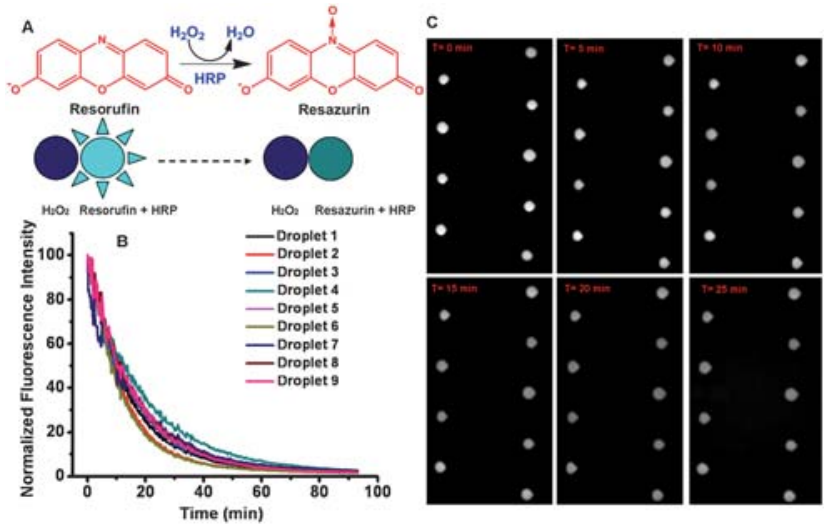

Fig. 4 (A) The schematic of hydrogen peroxide diffusion between two droplets and the oxidation of resorufin by HRP. (B) The time-course changes of fluorescence intensity in droplets containing $50 \mu \mathrm{M}$ resorufin and $0.07 \mathrm{mg} \mathrm{mL}^{-1} \mathrm{HRP}$. The $\left[\mathrm{H}_{2} \mathrm{O}_{2}\right]$ in the neighbouring droplet is $0.3 \mathrm{M}$. (C) The selected fluorescence microscopic pictures of the nine droplets showed the changes of fluorescence intensity in these droplets. Each fluorescent droplet containing resorufin and HRP was pressed and deformed by the trapped $\mathrm{H}_{2} \mathrm{O}_{2}$ droplet which was not visible in pictures. Oil phase: FC-77/1\% RainDance EA surfactant with flow rate $80 \mu \mathrm{L} \mathrm{h}^{-1}$. 
right droplet decreased, which was monitored to obtain the kinetic process of this diffusion-reaction model (Fig. 4A). To demonstrate the functionality of the traps, we selected 9 droplet pairs, and found that after $20 \mathrm{~min}$ the fluorescence intensities of the resorufin-containing droplets decreased to around $30 \%$, and levelled out at around $2 \%$ after $60 \mathrm{~min}$ (Fig. 4B). In future experiments, a motorised stage will be used to scan more droplet pairs. The fluorescence pictures showed that the sizes and shapes of droplets did not change significantly during this process, which indicated that the decrease of the fluorescence intensities should be attributed to $\mathrm{H}_{2} \mathrm{O}_{2}$ transfer and the initiated enzymatic reaction (Fig. 4C). Although droplets in different parts of the device might experience small difference in pressure, the kinetic curves obtained from different groups of droplets were very homogenous with a standard error around 5\% (Fig. 4c).

Because our system involves two kinetic processes, the fluorescence intensity curves are a combination of the kinetics of $\mathrm{H}_{2} \mathrm{O}_{2}$ diffusion across the interface as well as the enzymatic reaction. To understand the effect of the concentration of $\mathrm{H}_{2} \mathrm{O}_{2}$ on this rate, we changed $\left[\mathrm{H}_{2} \mathrm{O}_{2}\right]$ to compare the differences of the initial reaction rates. For each experiment, we determined the mean changes in fluorescence intensities by averaging the changes from at least 9 different droplets (ESI Fig. SI $2 \dagger$ ). As shown in Fig. 5A the higher $\left[\mathrm{H}_{2} \mathrm{O}_{2}\right]$, the faster the drop in fluorescence intensity. For comparison, the HRP catalyzed oxidation of resorufin by $\mathrm{H}_{2} \mathrm{O}_{2}$ in a 96 plate-well format proceeds much more rapidly (500 s, $80 \%$ conversion) for $\left[\mathrm{H}_{2} \mathrm{O}_{2}\right]$ ranging from $0.1 \mathrm{mM}$ to $1.0 \mathrm{mM}$ (ESI Fig. SI3†). Fig. 5B shows the maximum rate of drop in fluorescence intensity plotted against $\left[\mathrm{H}_{2} \mathrm{O}_{2}\right]$. Clearly, in droplets the apparent rate increases even at concentrations over $0.5 \mathrm{M}$ (50 times higher than 'bulk' concentration tested). This clearly indicates that in the droplet-based diffusionreaction system, the diffusion process was much slower than the enzymatic reaction rate.

In summary, we present here a new approach for the efficient trapping of droplet pairs and the study of molecular communication between droplets. The modular devices produced alternating (AB) droplet pairs with around $73 \%$ efficiency. The droplets were clearly deformed, strongly suggesting the formation of a surfactant bilayer between the trapped droplets, and thus providing a high throughput and effective platform for the observation and detection of diffusion across such bilayers. As a proof of principle, the oxidation of resorufin by hydrogen peroxidase was chosen as a model diffusion-reaction system and
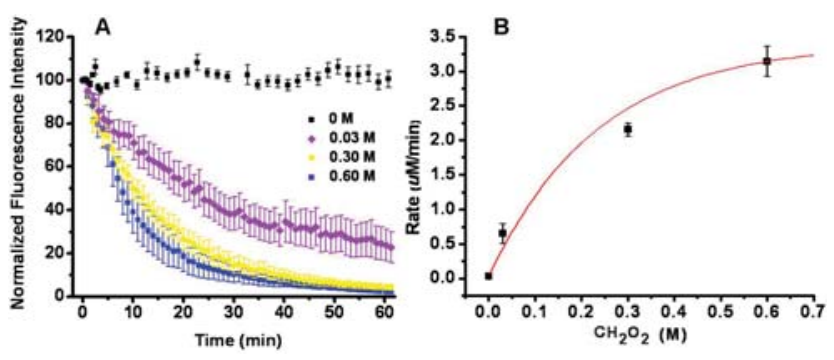

Fig. 5 (A) The kinetic curves of the fluorescence changes in droplets due to the diffusion of different $\left[\mathrm{H}_{2} \mathrm{O}_{2}\right](0 \mathrm{M}, 0.03 \mathrm{M}, 0.3 \mathrm{M}, 0.6 \mathrm{M})$ across droplets. (B) The initial respective rates of these enzymatic reactions were calculated from the kinetic curves in A. the results demonstrated hydrogen peroxide can transfer across droplets and initiate an enzymatic reaction. The results suggested that the diffusion of $\mathrm{H}_{2} \mathrm{O}_{2}$ between droplets is the key process to determine the total kinetic rate, and increasing the concentration of hydrogen peroxide can effectively increase the total kinetic rate of the model system. Experiments using phospholipids as surfactants to form lipid bilayers in microfluidics are being performed in our group, which opens up new experiments including biological systems.

\section{Acknowledgements}

Y. Bai is grateful for the CSC-Cambridge scholarship for financial support. $\mathrm{AH}$ was supported by an EU Marie-Curie fellowship (FP6 Early stage Training site ChemBioCam). This work was funded by the RCUK Basic Technology Programme. We thank Darren Link at RainDance Technologies for the gift of EA surfactant.

\section{Notes and references}

1 H. Song, D. L. Chen and R. F. Ismagilov, Angew. Chem., Int. Ed., 2006, 45, 7336-7356.

2 S.-Y. Teh, R. Lin, L.-H. Huang and A. P. Lee, Lab Chip, 2008, 8, 198 220; Y. Schaerli and F. Hollfelder, Mol. BioSyst., 2009, 5, 1392-1404.

3 E. Brouzes, M. Medkova, N. Savenelli, D. Marran, M. Twardowski, J. B. Hutchison, J. M. Rothberg, D. R. Link, N. Perrimon and M. L. Samuels, Proc. Natl. Acad. Sci. U. S. A., 2009, 106, 14195 14200; H. N. Joensson, M. L. Samuels, E. Brouzes, M. Medkova, M. Uhlen, D. R. Link and H. Andersson-Svahn, Angew. Chem., Int. Ed., 2009, 48, 2518-2521.

4 A. Huebner, L. F. Olguin, D. Bratton, G. Whyte, W. T. S. Huck, A. J. de Mello, J. B. Edel, C. Abell and F. Hollfelder, Anal. Chem., 2008, 80, 3890-3896.

5 J. Shim, L. F. Olguin, G. Whyte, D. Scott, A. Babtie, C. Abell, W. T. S. Huck and F. Hollfelder, J. Am. Chem. Soc., 2009, 131, 15251-15256.

6 L. Mazutis, J. C. Baret, P. Treacy, Y. Skhiri, A. F. Araghi, M. Ryckelynck, V. Taly and A. D. Griffiths, Lab Chip, 2009, 9, 2902-2908; S. Koster, F. E. Angile, H. Duan, J. J. Agresti, A. Wintner, C. Schmitz, A. C. Rowat, C. A. Merten, D. Pisignano, A. D. Griffiths and D. A. Weitz, Lab Chip, 2008, 8, 1110-1115.

7 F. Courtois, L. F. Olguin, G. Whyte, A. B. Theberge, W. T. S. Huck, F. Hollfelder and C. Abell, Anal. Chem., 2009, 81, 3008-3016.

8 G. Maglia, A. J. Heron, W. L. Hwang, M. A. Holdern, E. Mikhailova, Q. Li, S. Cheley and H. Bayley, Nat. Nanotechnol., 2009, 4, 437-440.

9 M. A. Holden, D. Needham and H. Bayley, J. Am. Chem. Soc., 2007, 129, 8650-8655.

10 H. Bayley, B. Cronin, A. Heron, M. A. Holden, W. L. Hwang, R. Syeda, J. Thompson and M. Wallace, Mol. BioSyst., 2008, 4, 1191-1208.

11 B. Zheng, J. D. Tice, L. S. Roach and R. F. Ismagilov, Angew. Chem., Int. Ed., 2004, 43, 2508-2511.

12 C. Holtze, A. C. Rowat, J. J. Agresti, J. B. Hutchison, F. E. Angile, C. H. J. Schmitz, S. Koster, H. Duan, K. J. Humphry, R. A. Scanga, J. S. Johnson, D. Pisignano and D. A. Weitz, Lab Chip, 2008, 8, 1632-1639.

13 T. Thorsen, R. W. Roberts, F. H. Arnold and S. R. Quake, Phys. Rev. Lett., 2001, 86, 4163-4166; M. Joanicot and A. Ajdari, Science, 2005, 309, $887-888$.

14 D. R. Link, S. L. Anna, D. A. Weitz and H. A. Stone, Phys. Rev. Lett., 2004, 92, 054503

15 L. M. Fidalgo, C. Abell and W. T. S. Huck, Lab Chip, 2007, 7, 984 986; C. Priest, S. Herminghaus and R. Seemann, Appl. Phys. Lett., 2006, 89, 134101; L. Mazutis, J.-C. Baret and A. D. Griffiths, Lab Chip, 2009, 9, 2665-2672.

16 K. Ahn, J. Agresti, H. Chong, M. Marguez and D. A. Weitz, Appl. Phys. Lett., 2006, 88, 264105. 
17 L. M. Fidalgo, G. Whyte, D. Bratton, C. F. Kaminski, C. Abell and W. T. S. Huck, Angew. Chem., Int. Ed., 2008, 47, 2042-2045.

18 A. Huebner, D. Bratton, G. Whyte, M. Yang, A. J. de Mello, C. Abell and F. Hollfelder, Lab Chip, 2009, 9, 692-698.

19 F. Courtois, L. F. Olguin, G. Whyte, D. Bratton, W. T. S. Huck, C. Abell and F. Hollfelder, ChemBioChem, 2008, 9, 439-446.

20 C. H. Schmitz, A. C. Rowat, S. Koster and D. A. Weitz, Lab Chip, 2009, 9, 44 49; W. W. Shi, J. H. Qin, N. N. Ye and B. C. Lin, Lab Chip, 2008, 8, 1432-1435; J. U. Shim, G. Cristobal, D. R. Link, T. Thorsen, Y. W. Jia, K. Piattelli and S. Fraden, J. Am. Chem. Soc., 2007, 129, 8825-8835.

21 D. C. Duffy, J. C. McDonald, O. J. A. Schueller and G. M. Whitesides, Anal. Chem., 1998, 70, 4974- 4984.

22 Y. Xia and G. M. Whitesides, Annu. Rev. Mater. Sci., 1998, 28, 153-184.

23 S. L. Anna, N. Bontoux and H. A. Stone, Appl. Phys. Lett., 2003, 82, 364-366.

24 J.-C. Baret, F. Kleinschmidt, A. E. Harrak and A. D. Griffiths, Langmuir, 2009, 25, 6088-6093.

25 A. M. Skelley, O. Kirak, H. Suh, R. Jaenisch and J. Voldman, Nat. Methods, 2009, 6, 147-152.
26 K. Funakoshi, H. Suzuki and S. Takeuchi, Anal. Chem., 2006, 78, 8169-8174.

27 N. Malmstadt, M. A. Nash, R. F. Purnell and J. J. Schmidt, Nano Lett., 2006, 6, 1961-1965.

28 K. Kato, P. Walde, N. Koine, S. Ichikawa, T. Ishikawa, R. Nagahama, T. Ishihara, T. Tsujii, M. Shudou, Y. Omokawa and T. Kuroiwa, Langmuir, 2008, 24, 10762-10770.

29 T. C. Logan, D. S. Clark, T. B. Stachowiak, F. Svec and J. M. J. Frechet, Anal. Chem., 2007, 79, 6592-6598.

30 H. J. Kim, J. Q. Boedicker, J. W. Choi and R. F. Ismagilov, Proc. Natl. Acad. Sci. U. S. A., 2008, 105, 18188-18193.

31 J. Q. Boedicker, M. E. Vincent and R. F. Ismagilov, Angew. Chem., Int. Ed., 2009, 48, 5908-5911.

32 S. Santesson, I. B.-R. Ramirez, P. Viberg, B. Jergil and S. Nilsson, Anal. Chem., 2004, 76, 303-308.

33 G. P. Bienert, A. L. B. Moller, K. A. Kristiansen, A. Schulz, I. M. Moller, J. K. Schjoerring and T. P. Jahn, J. Biol. Chem., 2006, 282, 1183-1192.

34 M. Zhou, Z. Diwu, N. Panchuk-Voloshina and R. P. Haugland, Anal. Biochem., 1997, 253, 162-168. 